Katarína Janková, Department of Mathematics, Comenius University, 84248 Bratislava, Slovakia. e-mail: jankova@fmph.uniba.sk

\title{
POINTS GENERATING THE PRINCIPAL MEASURE OF CHAOS
}

\begin{abstract}
The principal measure of chaos of a continuous map of an interval is generated by a pair of points at least one of which belongs to an omega limit set of the second kind if such set exists, or is zero in the case that such set does not exist.
\end{abstract}

For $f: I \rightarrow I$ continuous and $I$ a real compact interval let $f^{i}$ denote the $i$ th iterate of $f$. The sequence $\left\{f^{i}(x)\right\}_{i=0}^{\infty}$ is called the trajectory of the point $x$ and the set $\operatorname{Orb}(J)=\bigcup_{i=0}^{\infty} f^{i}(J)$ is the orbit of $J$ for any set $J \subset I$. It is periodic if for some nonnegative integer $n, f^{n}(J)=J$. In the case that $J=\{x\}, x$ is a periodic point of $f$. For any $x \in I, \omega(x)$ is the $\omega$-limit set, that is, the set of all limit points of the trajectory $\left\{f^{i}(x)\right\}_{i=0}^{\infty}$. There are several definitions of chaos for a dynamical system generated by $f$. We are going to discuss some features of distributional chaos introduced in [7] and studied in [5] and [6]. It is based on the behavior of upper and lower distribution functions defined as follows.

For any sequences of real numbers $\left\{a_{n}\right\}$ and $\left\{b_{n}\right\}$, where $a_{n}, b_{n} \in I$, denote

$$
\begin{aligned}
& \xi_{a_{n}, b_{n}}(t)=\liminf \frac{1}{n} \#\left\{i<n: / a_{i}-b_{i} /<t\right\} \\
& \xi_{a_{n}, b_{n}}^{*}(t)=\lim \sup \frac{1}{n} \#\left\{i<n: / a_{i}-b_{i} /<t\right\}
\end{aligned}
$$

\footnotetext{
Key Words: periodic point, $\omega$-limit set, principal measure of chaos

Mathematical Reviews subject classification: Primary 26A18, 58F08; Secondary 58F13, $54 \mathrm{H} 20$

Received by the editors April 10, 2000

*This research was supported by the Grant Agency of the Slovak Republic, Grant No. 1/4323/97. The author thanks J. Smítal for helpful discussion during the preparation of the paper.
} 
Since both $\xi_{a_{n}, b_{n}}$ and $\xi_{a_{n}, b_{n}}^{*}$ are nondecreasing (hence measurable), $0 \leq$ $\xi_{a_{n}, b_{n}}(t) \leq \xi_{a_{n}, b_{n}}^{*}(t) \leq|I|$, where $|I|$ denotes the length of interval $I$, we can consider

$$
\mu\left(a_{n}, b_{n}\right)=\frac{1}{|I|} \int_{0}^{|I|} \xi_{a_{n}, b_{n}}^{*}(t)-\xi_{a_{n}, b_{n}}(t) d t
$$

which is a number in $[0,1]$. Let $\mu(f, x, y)=\mu\left(f^{n}(x), f^{n}(y)\right)$. Then the principal measure of chaos is the number

$$
\mu(f)=\sup _{x, y \in I} \mu(f, x, y) .
$$

The function $f$ is distributionally chaotic if $\mu(f)>0$, otherwise it is nonchaotic. Furthermore, in compliance with [7], we shall set $F_{x, y}(t)=\xi_{f^{n}(x), f^{n}(y)}(t)$ and $F_{x, y}^{*}(t)=\xi_{f^{n}(x), f^{n}(y)}^{*}(t)$.

Properties of $\omega$-limit sets and their classification by Sharkovsky will be important in what follows. For basic properties of $\omega$-limit sets see [4]. Let $\omega(f)$ be the system of all $\omega$-limit sets of $f$. For any $\omega \in \omega(f), \omega$ is a closed nonempty subset of $I$ and $f(\omega)=\omega$. Consider the Hausdorff metric defined on $\Im x \Im$, where $\Im$ is the space of all closed subsets of $I$, in the following way. For any $A, B \in \Im$ let

$$
h(A, B)=\sup \{d(x, B) ; x \in A\}
$$

where

$$
d(x, B)=\inf \{|x-y| ; y \in B\}
$$

and put

$$
H(A, B)=\max \{h(A, B), h(B, A)\} .
$$

Then $H$ is the Hausdorff metric and the metric space $(\Im, H)$ is compact. In [3] it was also shown that $\omega(f)$ equipped with the Hausdorff metric is a compact subspace of $(\Im, H)$. Furthermore (see [3]), for any nondecreasing sequence $\left\{\omega_{n}\right\}_{n=1}^{\infty}$ of $\omega$-limit sets the closure of their union is also an $\omega$-limit set. An $\omega$-limit set is maximal if it is not properly contained in any other $\omega$-limit set. A maximal $\omega$-limit set is of the second kind if it contains a periodic point and it is infinite; otherwise, it is a maximal $\omega$-limit set of the first kind. An $\omega$-limit set $\omega$ has a periodic decomposition of period $k$ if there is a compact periodic interval $J \subset I$ of period $k$ such that Orb $(J) \supset \omega$ and such that for $0 \leq i \leq k$ the convex hulls of the set $P_{i}=f^{i}(J) \cap \omega$ are nonoverlapping; that is, they 
have at most one endpoint in common. Sets $P_{i}$ are called periodic portions of $\omega$. Periodic portion $P_{i}=f^{i}(J) \cap \omega$ is minimal if there is no periodic interval properly contained in $J$ such that its orbit contains $\omega$. An $\omega$-limit set can be finite - then it is an orbit of a periodic point - or infinite. The following theorem is a restatement of results from [7] concerning the characterization of infinite $\omega$-limit sets that will be important for our considerations.

Theorem 1. Let $A_{1}(f), A_{2}(f)$ be the classes of infinite maximal $\omega$-limit sets of $f$ of the first and second kind, respectively. Let $\omega(x)$ be an infinite $\omega$-limit set.

(i) If $\omega(x)$ has no minimal periodic portions, then $\omega(x)$ has periodic decompositions of arbitrarily high period.

(ii)If $\omega(x)$ has a minimal periodic portion, then $\omega(x) \subset \omega$ for some $\omega \in$ $A_{2}(f)$.

(iii) If $\omega_{1} \in A_{1}(f)$ and $\omega_{1} \neq \omega$ for some $\omega \in A_{1}(f) \cup A_{2}(f)$, then $\omega_{1} \cap \omega=\emptyset$. In particular, two distinct periodic portions of sets in $A_{1}(f)$ with the same period have no common points.

(iv) The intersection of any two distinct sets in $A_{2}(f)$ is finite, and if nonempty, it is the orbit of a periodic point. More precisely, if $P_{1}$ and $P_{2}$ are distinct periodic portions of any two sets in $A_{2}(f)$, and if $U=\operatorname{conv}\left(P_{1}\right)$ and $V=\operatorname{conv}\left(P_{2}\right)$, then $U \cap V=\emptyset$, or $U$ and $V$ have just one point in common, or $U \subset \operatorname{int}(V)$ or $V \subset \operatorname{int}(U)$.

(v) Let $\omega \in A_{2}(f)$. Then there is a minimal compact periodic interval $U$ of period $m \geq 1$ such that $\operatorname{Orb}(U) \supset \omega$. Moreover, if $J, K$ are compact intervals such that $J \cap \omega$ is infinite and $K \subset \operatorname{int}(U)$, then $f^{i+j m}(J) \supset K$ for some $i$ and any sufficiently large $j$. This situation is described by saying that $f \mid \omega$ is strongly transitive in $U$.

By applying mentioned results of [3] and [7], any $\omega$-limit set is contained in some maximal $\omega$-limit set. Furthermore, in [7], it was shown that if $x, y$ are such that $\omega(x), \omega(y)$ are contained in finite or infinite maximal sets of the first kind, then $\mu(f, x, y)=0$. Thus, in the case that $f$ has no infinite set of the second kind, $\mu(f)=0$. Our main aim is to show

Theorem 2. For any continuous $f: I \rightarrow I$ there are points $x_{0}, y_{0} \in I$ such that

$$
\mu(f)=\mu\left(f, x_{0}, y_{0}\right) .
$$

We shall consider sequences $\left\{x_{n}\right\},\left\{y_{n}\right\}$ of points in $I$ such that

$$
\mu(f)=\lim \mu\left(f, x_{n}, y_{n}\right)
$$

and $\left\{\mu\left(f, x_{n}, y_{n}\right)\right\}$ is nondecreasing. With respect to mentioned results $([7])$, if $\mu(f)>0$, the sequence $\left\{x_{n}\right\}$ can be chosen so that for any $n, \omega\left(x_{n}\right)$ is 
contained in some maximal $\omega$-limit set of the second kind $\omega_{n}$. Moreover, (see for example [7]), we can assume that the points $x_{n}$ belong to this $\omega$-limit set because for any $z \in I$ and for any $t \in I$

$$
F_{x_{n} z}(t)=F_{u z}(t) \text { for some } u \in \omega_{n}
$$

Let $\delta>0$ be such that $\mu(f)>\delta$. The next lemma shows that sequences $\left\{x_{n}\right\},\left\{y_{n}\right\}$ and maximal $\omega$-limit sets $\omega_{n}$, can be chosen such that for any $n$ there is a periodic portion of $\omega_{n}$ of length greater than $\delta / 2$.

Lemma 3. Let $\omega, \omega^{1}$ be maximal $\omega$ limit sets with periodic portions $P_{0} \ldots P_{k-1}$ and $P_{0}^{1} \ldots P_{s-1}^{1}$ respectively. If $\operatorname{diam} P_{i}<\delta$ and diam $P_{j}^{1}<\delta$ for any $i=0, \ldots, k-1$ and $j=0, \ldots, s-1$, then

$$
\mu(f, x, y)<2 \delta
$$

for any $x \in \omega, y \in \omega^{1}$.

Proof. Take $x \in \omega, y \in \omega^{1}$. The statement is evident if $k=1$ and $s=1$ since $\mu(f, x, y) \leq \frac{1}{|I|} \int_{0}^{|I|} F_{x y}^{*}(t)-F_{x y}(t) d t<2 \delta$. If $k>1$ or $s>1$, take $m=k s$ and consider $g=f^{m}$. We show that

$$
F_{x y}^{*}(t) \leq \frac{1}{m} \sum_{j=0}^{m-1} \xi_{g^{n}\left(f^{j}(x)\right), g^{n}\left(f^{j}(y)\right)}^{*}(t)
$$

which together with the fact that $\mu\left(g^{n}\left(f^{j}(x)\right), g^{n}\left(f^{j}(y)\right)\right)<2 \delta$ implies the result. Denote

$$
\begin{aligned}
A_{n}(t) & =\#\left\{0 \leq i<n: / f^{i}(x)-f^{i}(y) /<t\right\} \\
A_{n j}(t) & =\#\left\{0 \leq i<n: j \equiv i \quad(\bmod m) \text { and }\left|f^{i}(x)-f^{i}(y)\right|<t\right\}
\end{aligned}
$$

We have $A_{n}(t)=\bigcup_{j=0}^{m-1} A_{n j}(t)$ and $\# A_{n}(t) \leq \sum_{j=0}^{m-1} \# A_{n j}(t)$. For any real number $a$ let $[a]$ denote the integer part of $a$. It is easy to see that $\# A_{n j}(t) \leq \# B_{n j}(t)$, where

$$
B_{n j}(t)=\left\{k m+j: k=0,1 \ldots,\left[\frac{n}{m}\right] \text { and } / g^{k}\left(f^{j}(x)\right)-g^{k}\left(f^{j}(y)\right) /<t\right\} .
$$


We obtain

$$
\begin{aligned}
F_{x y}^{*}(t) & =\limsup \frac{1}{n} \# A_{n}(t) \leq \limsup \frac{1}{n} \sum_{j=0}^{m-1} A_{n j}(t) \\
& \leq \sum_{j=0}^{m-1} \lim \sup \frac{1}{n} \# B_{n j}(t) \leq \frac{1}{m} \sum_{j=0}^{m-1} \lim \sup \frac{1}{\left[\frac{n}{m}\right]} \# B_{n j}(t) \\
& \leq \frac{1}{m} \sum_{j=0}^{m-1} \xi_{g^{n}\left(f^{j}(x)\right), g^{n}\left(f^{j}(y)\right)}^{*}(t)
\end{aligned}
$$

Lemma 4. The number of $\omega$-limit sets of the second kind possessing periodic portion of diameter $\geq \delta$ is finite.

Proof. Follows immediately from Theorem 1.

The following theorem is a modification of Lemma 5.2 from [6].

Theorem 5. Let $f \in C(I, I)$, $\omega$ be a maximal $\omega$-limit set of the second kind, $f \mid \omega$ strongly transitive in $\operatorname{int} U, U$ a periodic interval and $p \in I$ a periodic point. Then for any sequence $\left\{x_{n}\right\}$, where $x_{n} \in \operatorname{int} \omega$ there is $x \in \omega$ with

$$
F_{x p} \leq \inf _{n} F_{x_{n} p} \text { and } F_{x p}^{*} \geq \sup _{n} F_{x_{n} p}^{*}
$$

Proof. The monotonicity of $F_{x_{n} p}$ and $F_{x_{n} p}^{*}$ enables us to take a countable set $T, T$ dense in $I$ and such that both $F_{x_{n} p}, F_{x_{n} p}^{*}$ are continuous at any $t \in T$ for any $n \in N$. Let $\left\{t_{j}\right\}$ and $\{x(j)\}$ be sequences of points from $T$ and $\left\{x_{n}\right\}$ respectively, such that for any $t \in T$ and any $x_{n}, t=t_{j}$ and $x_{n}=x_{j}$ for infinitely many $j$. Using induction we construct a sequence of positive integers

$$
k(1)<q(1)<k(2)<q(2)<\ldots<k(i)<q(i)<\ldots
$$

and a decreasing sequence of compact intervals $\left\{U_{i}\right\}_{i=1}^{\infty}$ with $\lim \operatorname{diam} U_{i}=0$ and such that for any $x \in U_{n}$ and $j \leq n$

$$
\begin{aligned}
& \frac{1}{k(j)} \#\left\{0 \leq i<k(j): / f^{i}(x)-f^{i}(p) /<t_{j}\right\} \leq F_{x(j) p}\left(t_{j}\right)+\frac{1}{j} \\
& \frac{1}{q(j)} \#\left\{0 \leq i<q(j): / f^{i}(x)-f^{i}(p) /<t_{j}\right\} \geq F_{x(j) p}^{*}\left(t_{j}\right)-\frac{1}{j}
\end{aligned}
$$


Take $U_{1}=U, k(1)=1, q(1)=2$ and assume that $U_{n}, k(n), q(n)$ have been defined such that $f^{j}\left(U_{n}\right) \cap \omega$ is infinite whenever $j$ is sufficiently large. Since both $U$ and $p$ are periodic and $f \mid \omega$ is strongly transitive in int $U$ there is some $m>q(n)$ such that $x(n+1) \in f^{m}\left(U_{n}\right)$ and $f^{m}(p)=p$. Let $a \in U_{n}$ such that $f^{m}(a)=x(n+1)$. Then $F_{a p}=F_{x(n+1) p}$ and $F_{a p}^{*}=F_{x(n+1) p}^{*}$. Now take as $U_{n+1} \subset U_{n}$ a compact neighborhood of $a$ with $\operatorname{diam} U_{n+1}<\frac{1}{2} \operatorname{diam} U_{n}$ such that (2), (3) hold for any $x \in U_{n+1}$. This is possible using the continuity of $F_{x_{n} p}$ and $F_{x_{n} p}^{*}$ at points of $T$ (see Lemma 5.1 in [7]). Similarly as in [7] $a, U_{n+1}$ can be chosen so that $f^{m}\left(U_{n+1}\right) \cap \omega$ is infinite. Now take $x \in \bigcap_{j=1}^{\infty} U_{j}$. For any $t \in T$ and $x_{n}$ take $j$ such that $t=t_{j}$ and $x_{n}=x(j)$. Since $x \in U_{j},(2)$ and (3) are satisfied. The fact that $j$ can be arbitrarily large implies $F_{x p}(t) \leq F_{x_{n} p}(t)$ and $F_{x p}^{*}(t) \geq F_{x_{n} p}^{*}(t)$ for any $t \in T$. However, $T$ is dense in $I$ which implies (1) is also satisfied for any $t \in I$. The argument that $x$ can be chosen in $\omega$ is the same as in the proof of Lemma 5.2 in [7].

Lemma 6. Let $\omega_{n}$ be a sequence of finite $\omega$-limit sets such that $\operatorname{diam} \omega_{n}>$ $D>0$ for all $n$. Assume that $\omega_{n}$ converges in the Hausdorff metric to an $\omega$ limit set contained in a maximal $\omega$-limit set of the second kind $\omega$. Let $\left\{p_{n}\right\}_{n=1}^{\infty}$ be a sequence of periodic points,$p_{n} \in \omega_{n}$. Then for any $\varepsilon>0$ and any $\delta>0$ there is $m_{0}$ such that for any $m \geq m_{0}$ there is a periodic point $q(m) \in \omega$ with $\#\left\{i<n: / f^{i}(q(m))-f^{i}\left(p_{m}\right) />\delta\right\}<n \varepsilon$ for any nonnegative integer $n$.

Proof. Using the Generalized specification property (GSP) from [2](stated also in [6]) for any $\varepsilon>0, \delta>0$ there is positive integer $K$ such that for any set $\left\{x_{1}, \ldots, x_{s}\right\}$ of $s \geq 1$ points in $\omega$ and any sequence

$$
0=k_{0}<k_{1}<\ldots<k_{s}
$$

of $s+1$ integers with $k_{i+1}-k_{i}>K$, there is a periodic point $u$ in $\omega$ with $f^{2 k_{s}}(u)=u$, and for all $x_{i}$ we have

$$
\begin{aligned}
& \#\left\{k_{i-1}<n \leq k \leq i: d\left(f^{n}(u), f^{n}\left(x_{i}\right)\right) \geq \delta\right\}<\left(k_{i}-k_{i-1}\right) \varepsilon \\
& \#\left\{k_{i-1}<n \leq k_{i}: d\left(f^{k_{s}+n}(u), f^{n}\left(x_{i}\right)\right) \geq \delta\right\}<\left(k_{i}-k_{i-1}\right) \varepsilon .
\end{aligned}
$$

Put $L=K+1$. By the uniform continuity of $f, f^{2}, \ldots, f^{L}$ there is $\delta_{1}<$ $\min \left\{\frac{\delta}{2}, \frac{D}{2}\right\}$ such that $|x-y|<\delta_{1}$ implies $\left|f^{i}(x)-f^{i}(y)\right|<\frac{\delta}{2}$ for $i=1,2, \ldots, L$. Because $\omega_{n}$ converges in the Hausdorff metric to a subset of $\omega$, there is $m_{0}$ such that the interval $\left(p_{m}-\delta_{1}, p_{m}+\delta_{1}\right)$ contains a point of $\omega$ for any $m \geq m_{0}$. Take $x_{1}$ as such a point. We have $\left|f^{L}\left(x_{1}\right)-f^{L}\left(p_{m}\right)\right|<\frac{\delta}{2}$. Further, there is a point from $\omega$ in $\left(f^{L}\left(p_{m}\right)-\delta_{1}, f^{L}\left(p_{m}\right)+\delta_{1}\right)$ - denote it as $x_{2}$. We have $\left|f^{L}\left(x_{2}\right)-f^{2 L}\left(p_{m}\right)\right|<\frac{\delta}{2}$. Let $t_{m}$ be the period of $p_{m}$. Similarly we construct points $x_{1}, x_{2}, \ldots, x_{r}$ where $r=\left[\frac{t_{m}}{L}\right]$, that is $t_{m}=r L+l, l \leq L$. Now we shall 
apply the GSP on $\left\{x_{1}, \ldots, x_{r}\right\}$ and sequence $k_{0}=0, k_{1}=L, k_{2}=2 L, \ldots, k_{r}=$ $r L+l$ so that there is $q(m) \in \omega$ such that $f^{2 k_{r}}(q(m))=q(m)$ and

$$
\begin{gathered}
\#\left\{i<n:\left|f^{i}(q(m))-f^{i}\left(p_{m}\right)\right|>\delta\right\} \\
=\sum_{j=0}^{r} \#\left\{k_{j} \leq i \leq k_{j+1}:\left|f^{i}(q(m))-f^{i}\left(p_{m}\right)\right|>\delta\right\}<n \varepsilon .
\end{gathered}
$$

Lemma 7. Let $\left\{a_{n}\right\},\left\{b_{n}\right\},\left\{c_{n}\right\}$ be arbitrary sequences of numbers in $I$ and $\varepsilon>0$. Assume that for any $n$

$$
\#\left\{i<n: b_{n} \neq c_{n}\right\}<n \varepsilon .
$$

Then for any $t \in R$, we have

$$
\xi_{a_{n}, b_{n}}(t)-\xi_{a_{n}, c_{n}}(t)<\varepsilon \text { and } \xi_{a_{n}, b_{n}}^{*}(t)-\xi_{a_{n}, c_{n}}^{*}(t)<\varepsilon .
$$

PROOF. $\xi_{a_{n}, b_{n}}(t)=\liminf \frac{1}{n} \#\left\{i<n:\left|a_{i}-b_{i}\right|<t\right\} \leq$

$\liminf \frac{1}{n}\left\{\#\left\{i<n:\left|a_{n}-c_{n}\right|<t\right.\right.$ and $\left.\left.b_{n}=c_{n}\right\}+n \varepsilon\right\} \leq$

$\leq \liminf \frac{1}{n} \#\left\{i<n:\left|a_{n}-c_{n}\right|<t\right\}+\varepsilon=\xi_{a_{n}, c_{n}}(t)+\varepsilon$.

The proof of the second part is similar.

Lemma 8. Let $x, y \in I$ be such that for any nonnegative integer $n, \mid f^{n}(x)-$ $f^{n}(y) \mid<\delta$. Then $|\mu(f, z, x)-\mu(f, z, y)|<2 \delta$ for any $z \in I$.

Proof. Since $\left|f^{n}(x)-f^{n}(z)\right|<t$ implies $\left|f^{n}(z)-f^{n}(y)\right|<t+\delta$ for any $t$, we obtain the inequalities $F_{z x}(t) \leq F_{z y}(t+\delta)$ and $F_{z x}^{*}(t) \leq F_{y z}^{*}(t+\delta)$. Using these we have

$$
\begin{aligned}
& \mu(f, z, x)=\frac{1}{|I|} \int_{-\infty}^{\infty} F_{z x}^{*}(t)-F_{z x}(t) d t \leq \frac{1}{|I|} \int_{-\infty}^{\infty} F_{z y}^{*}(t+\delta)-F_{z y}(t-\delta) d t= \\
& \mu(f, z, y)+\frac{1}{|I|} \int_{-\infty}^{\infty} F_{z y}(t+\delta)-F_{z y}(t-\delta) d t<\mu(f, z, y)+2 \delta . \\
& \text { Similarly, } \mu(f, z, y)<\mu(f, z, x)+2 \delta, \text { which implies the assertion. }
\end{aligned}
$$

Lemma 9. Let $\left\{a_{n}\right\},\left\{b_{n}\right\}$ and $\left\{c_{n}\right\}$ be arbitrary sequences of numbers in I with

$$
\#\left\{i<n:\left|b_{n}-c_{n}\right|>\delta\right\}<n \varepsilon \text {. Then }\left|\mu\left(a_{n}, b_{n}\right)-\mu\left(a_{n}, c_{n}\right)\right|<2 \delta+2 \varepsilon .
$$

Proof. Define $c_{n}^{\prime}=b_{n}$ if $\left|b_{n}-c_{n}\right| \geq \delta$ and $c_{n}^{\prime}=c_{n}$ if $\left|b_{n}-c_{n}\right|<\delta$. Then by our previous lemma we have $\left|\mu\left(a_{n}, b_{n}\right)-\mu\left(a_{n}, c_{n}^{\prime}\right)\right|<2 \delta$ and by Lemma $7,\left|\mu\left(a_{n}, c_{n}\right)-\mu\left(a_{n}, c_{n}^{\prime}\right)\right|<2 \varepsilon$. Thus we obtain $\left|\mu\left(a_{n}, b_{n}\right)-\mu\left(a_{n}, c_{n}\right)\right|<2 \delta+$ $2 \varepsilon$. 
Corollary 10. Let $\left\{\mu\left(f, x, p_{n}\right)\right\}_{n=1}^{\infty}$ be a nondecreasing sequence, $p_{n}$ a periodic point, $\operatorname{Orb}\left(p_{n}\right)=\omega_{n}$, where $\omega_{n}$ converges in the Hausdorff metric to an $\omega$-limit set contained in a maximal $\omega$-limit set of the second kind $\omega$. Then for any $n$ there is $q_{n} \in \omega$ such that $\sup \mu\left(f, x, p_{n}\right) \leq \sup \mu\left(f, x, q_{n}\right)$.

Proof. Let $m$ be any nonnegative integer. Take $\varepsilon, \delta$ such that $\varepsilon+\delta<\frac{1}{2 m}$. By Lemma 6 there is $n_{m}$ such that for some periodic point $q_{n_{m}} \in \omega$

$$
\#\left\{0 \leq i<n:\left|f^{i}\left(q_{n_{m}}\right)-f^{i}\left(p_{n_{m}}\right)\right|>\delta\right\}<\varepsilon n .
$$

Then by Lemma 9 ,

$$
\mu\left(f, x, p_{n_{m}}\right)<\mu\left(f, x, q_{n_{m}}\right)+\frac{1}{m} .
$$
ment.

Consequently, $\sup \mu\left(f, x, p_{n_{m}}\right) \leq \sup \mu\left(f, x, q_{n_{m}}\right)$, which implies the state-

Lemma 11. Let $\omega$ be an $\omega$-limit set with periodic decompositon of arbitrarily large period. Then for any $x \in I$ there is $y_{0} \in \omega$ such that $\mu\left(f, x, y_{0}\right)=$ $\sup _{y \in \omega} \mu(f, x, y)$.

Proof. Define $\varphi: \omega \rightarrow[0,1]$ by $\varphi(y)=\mu(f, x, y)$. By Lemma 8 and because of the decomposability of $\omega, \varphi$ is continuous on $\omega$, and since $\omega$ is compact it achieves its maximum.

Proof of Theorem 2. With respect to Lemma 3 and Lemma 4 we can assume that $\mu(f)=\lim \mu\left(f, x_{n}, y_{n}\right)$, where $x_{n} \in \omega, \omega$ is a maximal $\omega$-limit set of the second kind and $y_{n} \in \omega_{n}$, with $\omega_{n}$ a maximal $\omega$-limit set. We shall establish our result by considering the following cases:

Case I. $\omega_{n}$ is a periodic orbit.

Case Ia. diam Orb $y_{n} \rightarrow 0$. Then there is a fixed point $p$ so that $y_{n} \rightarrow p$. By Lemma 8 , for any $\delta>0$ there is $n_{0}$ such that for all $n \geq n_{0}, \mid \mu\left(f, x, y_{n}\right)-$ $\mu(f, x, p) \mid<\delta$. Then $\sup \mu\left(f, x, y_{n}\right) \leq \mu(f, x, p)$, and using Theorem 5 we obtain the result.

Case Ib. Assume that diam $\omega_{n}>\varepsilon$ for all $n$. Let $\omega_{n}$ converge in the Hausdorff metric to an $\omega$-limit set contained in $\omega$, a maximal omega limit set. We have to consider these possibilities: $\omega$ is a periodic orbit, an infinite set of the first kind or a maximal $\omega$-limit set of the second kind. The first possibility is solved in Ia., the second follows from Lemma 11 and the last follows from Lemma 5.2 of [7].

Case II. $\omega_{n}$ is a maximal $\omega$-limit set of the first kind for all $n$. In this case $\omega_{n}$ can be covered by a union of disjoint periodic intervals of diameter less 
than $\frac{1}{2^{n}}$, and let $p_{n}$ be a periodic point from this union. Then by Lemma 8 $\sup \mu\left(f, x, y_{n}\right)=\sup \mu\left(f, x, p_{n}\right)$ and the result follows from I.

Case III. $\omega_{n}$ is a maximal $\omega$-limit set of the second kind for all $n$ :

Case IIIa. For infinitely many $n$, diam $\omega_{n}>\varepsilon$. Then there is maximal $\omega$-limit set of the second kind $\omega^{*}$ such that $y_{n} \in \omega^{*}$ for infinitely many $n$ and again we can use Lemma 5.2 of [7] to obtain the result.

Case IIIb. diam $\omega_{n} \rightarrow 0$ for $n \rightarrow \infty$. Then with respect to Corollary 10 there are $p_{n}$ periodic points, $p_{n} \in \omega_{n}$ with $\sup \mu\left(f, x, y_{n}\right)=\sup \mu\left(f, x, p_{n}\right)$ and the problem reduces to case I.

Remark 1. The result does not hold for maps of the unit square. This follows from [1] where an example of a distributionally chaotic triangular map whose principal measure is not generated by a pair of points is given.

\section{References}

[1] M. Babilonová, Distributional chaos for triangular maps, Annales Mathematicae Silesianae, 13(1999), 33-38.

[2] F. Balibrea, B. Schweizer, A. Sklar and J. Smítal, Generalized specification property and distributional chaos, to appear.

[3] A. Block, A. M. Bruckner, P. D. Humke and J. Smítal, The space of $\omega$-limit sets of a continuous map of the interval, Trans. Amer. Math. Soc., 348(1996), 1357-1372.

[4] L. S. Block and W. A. Coppel, Dynamics in one dimension, Lecture Notes in Math. 1513, Springer, Berlin, 1992.

[5] Liao Gongfu and Fan Qinjie, Minimal subshifts which display SchweizerSmítal chaos and have zero topological entropy, Science in China, Ser.A 41(1998), 33-38.

[6] A. Sklar and J. Smítal, Distributional chaos on compact metric spaces via specification properties, Journ. Math. Anal. and Appl. 241(2000), 181188.

[7] B. Schweizer and J. Smítal, Measures of chaos and spectral decomposition of dynamical systems on the interval, Trans. Amer. Math. Soc., 344(1994), 737-754. 
